\title{
INVESTIGATION OF THE COMPOSITION OF COMPLEXES AND THE STOICHIOMETRY OF NON-COMPLEX REACTIONS BY FLOW INJECTION METHOD
}

\author{
IrNGFu Lu and HuICHANG MA* \\ Research Center for Eco-Environmental Sciences, Academia Sinica, P.O. Box 2871, Beijing 100085, China
}

(Received 2 December 1992. Revised 7 November 1992. Accepted 8 December 1992)

\begin{abstract}
Summary-Combining the Asmus straight-line method with the flow injection (FI) technique, a new FI-straight-line method is established to investigate the formulas of the blue, the purple and the red complexes formed by iron(III) and Tiron in different $\mathrm{pH}$ solutions. Using the proposed FI-straight-line method, the stoichiometries of cyanide and barbituric acid for producing the intermediate and the final product of the chromogenic reaction of cyanide with pyridine-barbituric acid reagent are also studied.
\end{abstract}

Various spectrophotometric methods have been established to investigate the empirical formulas of colored complexes in solution. Among them the Asmus straight-line' method has the advantages of being more suitable for determining the composition of weak complexes and applicable for complex systems where ligands are not pure and precise ligand concentration is not known.

The straight-line method is conventionally performed by a manual procedure which does not allow investigation of the composition of unstable products of intermediate of some complicated reactions. It has been adapted to a flow technique by Martinez et $a .^{2}$ The method is based on the merging of two solutions (metal and reagent), keeping the flow rate of one of them constant and increasing the other stepwise.

The flow injection (FI) technique has many advantages. One of them is that it allows fast reactions to be investigated. In the present study, the straight-line method was combined with the flow injection technique to measure complex formulas, determine the stoichiometry of non-complex reactions and to investigate the mechanism of complicated reactions.

\section{PRINCIPLE}

The straight-line method was initially established by Asmus to determine the composition of mononuclear complexes. Later, it was expanded to measure the composition of polynuclear complexes by Klausen et al. ${ }^{3}$ The

*To whom correspondence should be addressed. principle of the method can be expressed as follows.

For the reaction of the form

$$
m \mathrm{M}+n \mathrm{~L}=\mathrm{M} m \operatorname{Ln}(m, n \geqslant 1) .
$$

Constant volume of a standard solution of $M$ (concentration $C_{\mathrm{M}}^{0}$ ) and varying volumes $\left(V_{\mathrm{L}}\right)$ of a standard solution of $L$ (concentration $C_{\mathrm{L}}^{0}$ ) are added into a series of volumetric flasks of volume $V$, the $\mathrm{pH}$ is adjusted and an inert salt solution is added. Then the mixtures are diluted to volume and the absorbance $(A)$ is measured at a suitable wavelength. Assuming

$$
\begin{gathered}
{[\mathrm{M} m \mathrm{Ln}] \ll C_{\mathrm{L}}} \\
\epsilon_{\mathrm{M}}=\epsilon_{\mathrm{L}}=0
\end{gathered}
$$

where the brackets refer to the equilibrium concentration. $C$ to the analytical or total concentration and $\epsilon$ to molar absorptivity. Expressing $1 /\left(C_{\mathrm{L}}\right)^{n}$ graphically as a function of $1 / A$ for different sets of $n$, a straight line will be expected for the correct value of $\boldsymbol{n}$. Similarly, if a constant concentration of $L$ and different amounts of $M$ are applied, a straight line would be obtained for the correct value of $m$ by plotting $1 /\left(C_{\mathrm{M}}\right)^{m}$ against $1 / A$ for different sets of $m$. If the complex formed during the determination of $n$ is the same as that formed during the determination of $m$, the complex can be represented by $\mathrm{M} m \mathrm{~L} n$.

When the straight-line method was adapted for a flow injection system, the flow system can be designed as following: A carrier stream composed of a buffer and an unreacting electrolyte is propelled to the system to control the $\mathrm{pH}$ and the ionic strength of the system. Component $M$ 
is introduced into the system at a constant concentration. A series of $L$ are injected into the system as samples. The sample zone merges with reagent $\mathrm{M}$ to form the colour species and the peak absorbance $(A)$, corresponding to $C_{\mathrm{L}}^{\max }$ (the analytical concentration of $L$ at peak maximum), is detected.

In FIA, a chemical reaction is usually nonequilibrated. Namely,

$$
C_{\mathrm{MmL} n}^{\max } \leqslant[\mathrm{M} m \mathrm{~L} n]^{\max }
$$

in which $C_{\mathrm{MmLn}}^{\max }$ represents the peak maximum concentration of the produced $\mathrm{M} m \mathrm{~L} n$ when the peak absorbance $(A)$ is detected. $[\mathrm{MmLn}]^{\max }$ is the equilibrium concentration of $\mathrm{M} m \mathrm{~L} n$ at the peak maximum.

When

$$
[\mathrm{M} m \mathrm{~L} n]^{\max } \ll C_{\mathrm{L}}^{\max } .
$$

From equations (4) and (5), we have

$$
C_{\mathrm{MmL} n}^{\max } \ll C_{\mathrm{L}}^{\max } \text {. }
$$

Assuming

$$
\epsilon_{\mathrm{M}}=\epsilon_{\mathrm{L}}=0
$$

a straight line will be obtained for the correct value of $n$ if $1 /\left(C_{\mathrm{L}}^{\max }\right)^{n}$ is graphically expressed as a function of $1 / A$ for different sets of $n$.

If the volume and the concentration of the injected $\mathrm{L}$ are $S_{\mathrm{v}}$ and $C_{\mathrm{L}}^{0}$, respectively, then according to FIA theory, ${ }^{4}$

$$
C_{\mathrm{L}}^{\max }=C_{\mathrm{L}}^{0} / D_{\mathrm{L}}^{\mathrm{m}}=C_{\mathrm{L}}^{0} /\left(2 \pi^{3 / 2} R^{2} D_{\mathrm{f}}^{1 / 2} T^{1 / 2} / S_{\mathrm{v}}\right)
$$

where $D_{\mathrm{L}}^{\max }$ is the dispersion coefficient of $\mathrm{L}$ at peak maximum, $D_{\mathrm{f}}$ is the axial dispersion coefficient, $R$ is the tube radius and $T$ is the residence time. They are all constants for a certain manifold. Therefore,

$$
C_{\mathrm{L}}^{\max }=K C_{\mathrm{L}}^{0} S \mathrm{v}
$$

in which $K$ is a constant. If $S \mathrm{v}$ is kept constant, then

$$
\begin{aligned}
C_{\mathrm{L}}^{\max } & =K_{1} C_{\mathrm{L}}^{0} \\
1 /\left(C_{\mathrm{L}}^{\max }\right)^{n} & =K_{1}^{\prime} /\left(C_{\mathrm{L}}^{0}\right)^{n},
\end{aligned}
$$

where $K_{1}$ and $K_{1}^{\prime}$ are constants.

It is well known that $C_{\mathrm{L}}^{\max }$ will, for $D_{\mathrm{L}}^{\max } \geqslant 2$, increase linearly with increase of $S \mathrm{v}$. If $C_{\mathrm{L}}^{0}$ remains unchanged and $D_{\mathrm{L}}^{\max }$ is controlled in the region of $D_{\mathrm{L}}^{\max } \geqslant 2$, then from equation (8) we have

$$
\begin{aligned}
C_{\mathrm{L}}^{\max } & =K_{2} S \mathrm{v} \\
1\left(C_{\mathrm{L}}^{\max }\right)^{n} & =K_{2}^{\prime} /(S \mathrm{v})^{n} .
\end{aligned}
$$

According to equations (10) and (12), it is seen that a straight line will be obtained at the correct set of $n$ by plotting $1 /\left(C_{\mathrm{M}}^{0}\right)^{n}$ against $1 / A$ or by plotting $1 /(S v)^{n}$ against $1 / A$. Similarly, if various concentrations or volumes of $\mathbf{M}$ are injected and a constant concentration of $\mathrm{L}$ is pumped into the flow system, the value of $m$ can be obtained by plotting $1 /\left(C_{\mathrm{L}}^{0}\right)^{m}$ against $1 / A$ or by plotting $1 /(S \mathrm{v})^{m}$ against $1 / A$.

If $\epsilon_{M} \neq \epsilon_{L} \neq 0$, the absorbance of the excess ligand should be corrected. This can be done easily by the stopped-flow FIA technique if the reaction rate is not very fast. Unfortunately, the reaction of iron(III) and Tiron is too fast to use the stopped-flow FIA method. In the present study, absorbance was corrected simply by adjusting the baseline to $A=0$.

\section{EXPERIMENTAL}

\section{Reagents}

Unless otherwise stated, analytical reagents from Beijing Chemical Factory were used throughout.

Iron(III) solution: A $0.1000 M$ iron(III) stock solution was prepared by dissolving $0.5590 \mathrm{~g}$ of iron powder in $15 \mathrm{ml}$ of $6 \mathrm{M} \mathrm{HCl}$, adding $3 \mathrm{~g}$ of $\mathrm{KClO}_{3}$, and diluting to $100 \mathrm{ml}$ with water. Working solutions were made by diluting this stock solution with $0.1 M$ hydrochloric acid.

1,2-Dihydroxybenzen-3,5-disulfonate (trade name Tiron, chemical pure, Shanghai Chemical Reagent Factory) solution: A $5.0000 \times 10^{-2} M$ Tiron stock solution was prepared by dissolving $1.661 \mathrm{~g}$ of Tiron in $100 \mathrm{ml}$ of water. Working solutions were made by diluting this stock solution with water.

Pyridine solution: Add $12 \mathrm{ml}$ of concentrated hydrochloric acid in $60 \mathrm{ml}$ of pyridine, dilute to $250 \mathrm{ml}$ with water.

Pyridine-barbituric acid reagent: Place 8.205 $\mathrm{g}$ of barbituric acid in a $250 \mathrm{ml}$ standard flask and add just enough water to wash the flask wall and wet the barbituric acid. Add $60 \mathrm{ml}$ of pyridine and mix. Then $12 \mathrm{ml}$ of concentrated hydrochloric acid is added and mixed. Dilute to volume with water and mix when the mixture has cooled to room temperature. This stock contains $0.20 \mathrm{M}$ barbituric acid. Working solutions were prepared by diluting this stock with the above prepared pyridine solution.

Chloramine-T solution: Dissolve $0.5 \mathrm{~g}$ of chloramine- $\mathrm{T}$ in $100 \mathrm{ml}$ of water.

Standard cyanide solution: Standard cyanide solutions were prepared from a stock solution of potassium cyanide $\left(1000 \mu \mathrm{g} \mathrm{CN}^{-} / \mathrm{ml}, \mathrm{pH} 11\right)$ with $1 \mathrm{~g} / \mathrm{l}$ sodium hydroxide solution. 


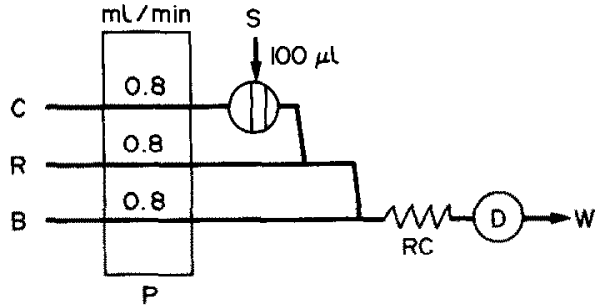

Fig. 1. Manifold used for determining formulas of complexes formed by iron(III) and Tiron in different $\mathrm{pH}$ solutions. C, water carrier; S, samples of Tiron or iron(III); $\mathrm{R}$, iron(III) or Tiron solution; $B$, buffer solutions of $\mathrm{pH} 4.0$, 5.9 or 9.0 ; RC, reaction coil (120 cm long, $0.7 \mathrm{~mm}$ i.d.); $D$, spectrophotometer, wavelength set at $620 \mathrm{~nm}, 560 \mathrm{~nm}$ or $480 \mathrm{~nm}$; P, peristaltic pump; W, waste.

Buffer solution of $\mathrm{pH}$ 4.0: Dissolve $7.94 \mathrm{~g}$ of anhydrous sodium acetate in $100 \mathrm{ml}$ of water, add $47 \mathrm{ml}$ of acetic acid and dilute to $500 \mathrm{ml}$ with water.

Buffer solution of pH 5.9: Dissolve $78 \mathrm{~g}$ of sodium dihydrogen phosphate in $1000 \mathrm{ml}$ of water, and adjust to $\mathrm{pH} 5.9$ with concentrated sodium hydroxide solution.

Buffer solution of $\mathrm{pH}$ 9.0: Dissolve $35 \mathrm{~g}$ of ammonium chloride in $100 \mathrm{ml}$ of water, add $24 \mathrm{ml}$ of ammonia solution (contains about $28 \%(\mathrm{w} / \mathrm{v})$ ammonia, and dilute to $500 \mathrm{ml}$ with water.

A FIA-91 flow injection analyzer (Shanghai No. 3 Analytical Instrument Factory) was used. This is an integrated instrument which consists of two 4-channel peristaltic pumps, a 16-port value, a grating spectrophotometric detector, a Laser PP40 4 color $X-Y$ printer plotter and a microcomputer system. Program control and data processing are conducted automatically by the microcomputer system.

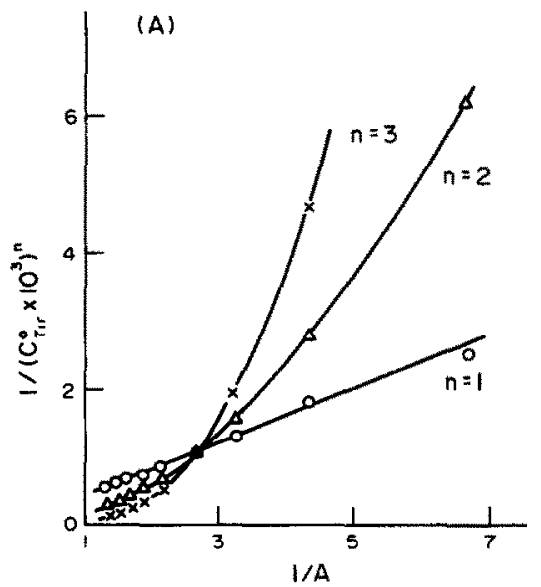

RESULTS AND DISCUSSION

\section{Determination of composition of $\mathrm{Fe}(I I I)-T i r o n$ complex}

Ferric ions produce three different colored complexes with Tiron, depending on the $\mathrm{pH}$ of the solution. According to Harrey et al., ${ }^{5}$ the colors of the three complexes are blue ( $\mathrm{pH}<5.6$ ), purple ( $\mathrm{pH} 5.7-6.9)$ and red $(\mathrm{pH}>7.0)$, and their metal-ligand molar ratios are 1:1,1:2 and 1:3, respectively. The present study is to investigate the composition of the three complexes by the proposed FI-straightline method.

The manifold depicted in Fig. 1 was designed to measure the formulas, represented by $\mathrm{Fe}_{m}(\mathrm{Tir})_{n}$, of the three complexes. In the first series of experiments, the blue complex formed at $\mathrm{pH} 4.0$ was investigated. To determine the value of $n$, a series of different concentrations of Tiron $\left(C_{\mathrm{Tir}}^{0}=0.8-2.0 \times 10^{-3} M\right)$ were injected into water carrier, while a constant concentration of iron(III) ion $\left(C_{\mathrm{Fe}}^{0}=1 \times 10^{-3} M\right)$ and a buffer of pH 4.0 were propelled into the flow system. Absorbance was detected at $620 \mathrm{~nm}$, the peak absorbance wavelength of the blue complex, and the results are shown in Fig. 2(A), which was obtained by plotting $1 /\left(C_{\mathrm{TIr}}^{0}\right)^{n}$ against $1 / A$ for different sets of $n$. The value of $m$ was determined similarly, the only difference being that the Tiron solution is introduced at a constant concentration $\left(C_{\mathrm{Tir}}^{0}=6 \times 10^{-3} \mathrm{M}\right)$ and various concentrations of ferric ion $\left(C_{\mathrm{Fe}}^{0}=\right.$ $0.6-1.8 \times 10^{-3} \mathrm{M}$ ) were injected into the system. The curves obtained for the determination of $m$ are shown in Fig. 2(B). Figure 2 indicates that a straight line occurs for $n=1$ or $m=1$. As

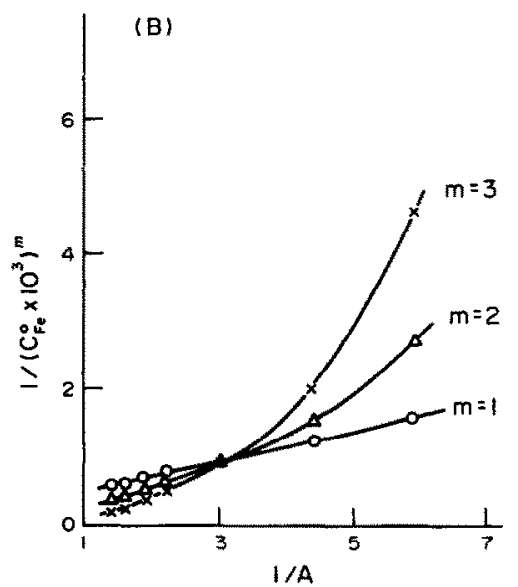

Fig. 2. The use of the FI-straight-line method for determining the formula of the blue complex formed by iron(III) and Tiron in a buffer solution of $\mathrm{pH} 4.0$ : (A) determination of $n, C_{\mathrm{Tu}}^{0}=0.8-2.0 \times 10^{-3} \mathrm{M}$, $C_{\mathrm{Fe}}^{0}=1.0 \times 10^{-3} \mathrm{M} ;(\mathrm{B})$ determination of $m, C_{\mathrm{Ttr}}^{0}=6.0 \times 10^{-3} \mathrm{M}, \mathrm{C}_{\mathrm{Fe}}^{0}=0.6-1.8 \times 10^{-3} \mathrm{M}$. 

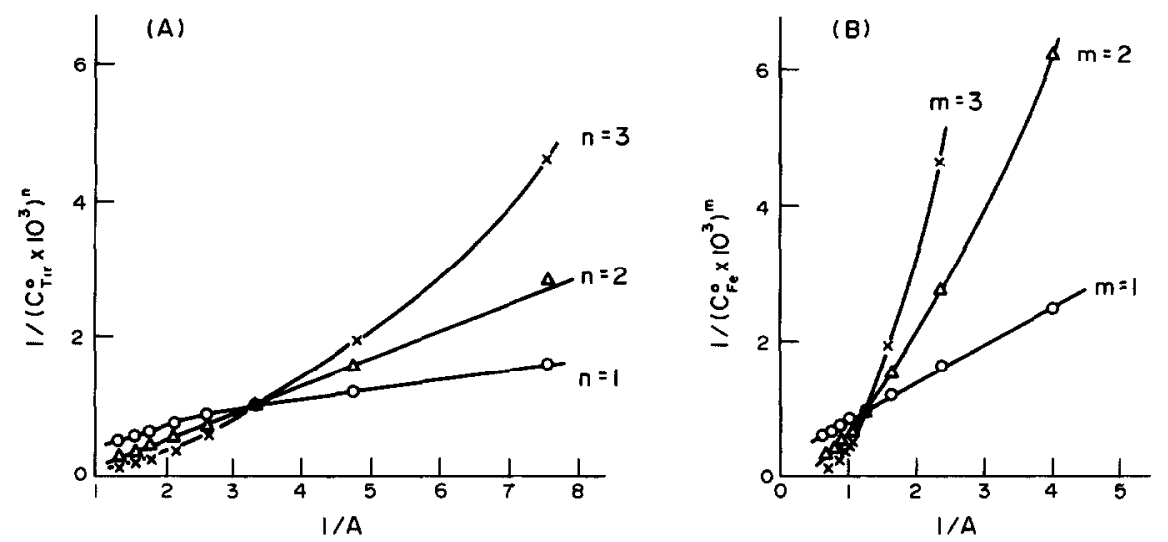

Fig. 3. The use of the FI-straight-line method for determining the formula of the purple complex formed by iron(III) and Tiron in a buffer solution of $\mathrm{pH} 5.9$ : (A) determination of $n, C_{\mathrm{Tr}}^{0}=0.6-2.0 \times 10^{-3} M$, $C_{\mathrm{Fe}}^{0}=1.0 \times 10^{-3} \mathrm{M}$; (B) determination of $m, C_{\mathrm{TI}}^{0}=6.0 \times 10^{-3} \mathrm{M}, C_{\mathrm{Fe}}^{0}=0.4-1.8 \times 10^{-3} \mathrm{M}$.

only one kind of complex is produced by iron(III) and Tiron in a buffer solution of $\mathrm{pH}$ $4.0,{ }^{5}$ the formula of the blue complex is Fe(Tir).

The compositions of the purple and the red complexes are measured similarly. For investigating the purple complex, a buffer of $\mathrm{pH} 5.9$ was adopted and the absorbance was detected at $560 \mathrm{~nm}$. Results are shown in Fig. 3. For producing the red complex, a buffer of $\mathrm{pH} 9.0$ was adopted. Absorbance was detected at 480 $\mathrm{nm}$ and the resulting curves are shown in Fig. 4. From Figs 3 and 4 it is seen that the compositions of the purple and the red complexes are $\mathrm{Fe}(\mathrm{Tir})_{2}$ and $\mathrm{Fe}(\mathrm{Tir})_{3}$, respectively.

The formulas of the blue, the purple and the red complex obtained above agree well with the metal-ligand molar ratios obtained by Harrey et $a .^{5}$ This fact indicates that the FI-straightline method is suitable for determining the composition of complex.

Reaction mechanism of cyanide with pyridinebarbituric acid

Cyanide is usually determined by the pyridine-barbituric acid method based on the Koning synthesis. Determination is conventionally conducted by detecting the final product of the reaction at $580 \mathrm{~nm}$. In our previous study, ${ }^{8}$ an intermediate of the reaction with a peak absorbance at $494 \mathrm{~nm}$ was found and was applied to determine cyanide successfully by FIA. In the present study, we measured the molar ratios of cyanide to barbituric acid for producing the intermediate and the final product, in order to propose a more detailed mechanism of the reaction.

This above-mentioned reaction is a complicated reaction, in which cyanide first reacts with chloramine- $\mathrm{T}$ or bromine water to produce cyanogen chloride or bromide. The produced cyanogen chloride or bromide quantitatively oxidizes pyridine to glutaconicaldehyde which condenses with barbituric acid to form a colored polymethine dye. The overall stoichiometry of cyanide and barbituric acid is $1: 2 .^{7}$ Unfortunately, the stoichiometry of cyanide and barbituric acid for producing the intermediate is not clear. Obviously, a more detailed reaction
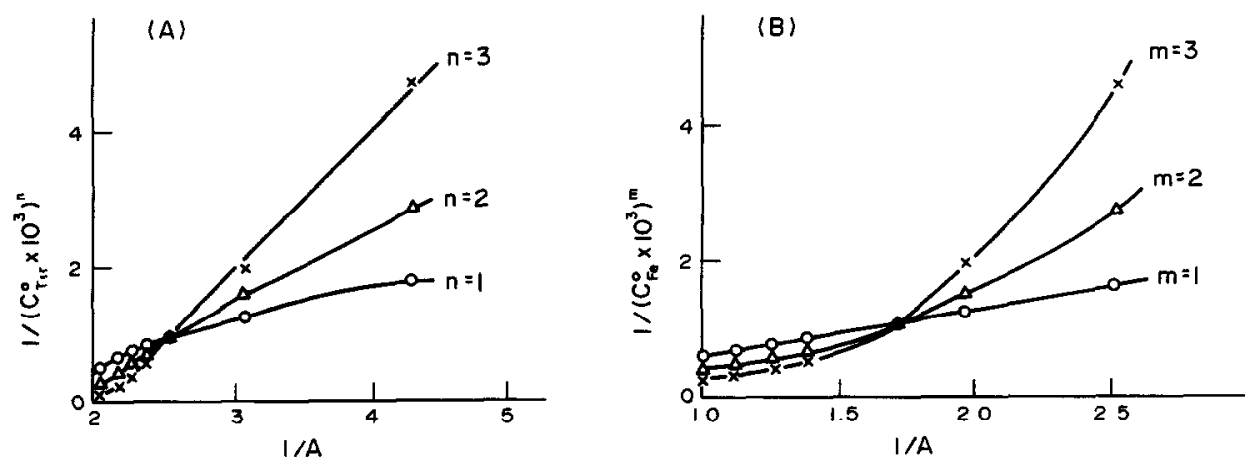

Fig. 4. The use of the FI-straight-line method for determining the formula of the red complex formed by iron(III) and Tiron in a buffer solution of $\mathrm{pH} 9.0$ : (A) determination of $n, C_{\mathrm{Tir}}^{0}=0.6-1.8 \times 10^{-3} M$, $C_{\mathrm{Fe}}^{0}=1.0 \times 10^{-3} \mathrm{M}$; (B) determination of $m, C_{\mathrm{Tur}}^{0}=6.0 \times 10^{-3} \mathrm{M}, C_{\mathrm{Fe}}^{0}=0.6-1.8 \times 10^{-3} \mathrm{M}$. 


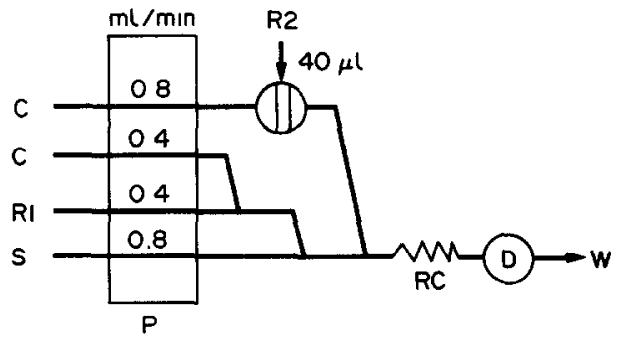

(A)

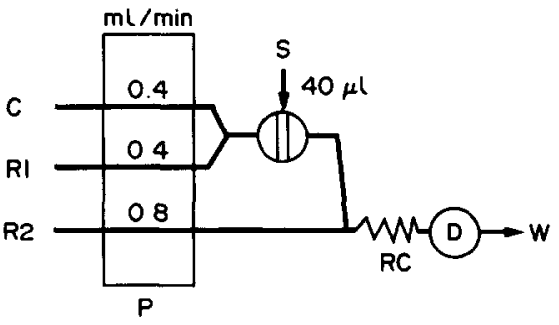

(B)

Fig. 5. Manifold used for investigating the stoichiometry of cyanide and barbituric acid during the formation of the intermediate and the final product: (A) determination of $n$; (B) determination of $m$; C, sodium dihydrogen phosphate buffer solution ( $\mathrm{pH}$ 5.9): R1, chloramine-T solution; R2, pyridinebarbituric acid reagent; S, cyanide; $R C$, reaction coil $(30 \mathrm{~cm}$ long, $0.7 \mathrm{~mm}$ i.d. or $270 \mathrm{~cm}$ long, $0.7 \mathrm{~mm}$

i.d.); D, spectrophotometer, wavelength set at $494 \mathrm{~nm}$ or $580 \mathrm{~nm}$; P, peristaltic pump, W, waste.

mechanism could be acquired if the stoichiometry is determined. The stoichiometry of cyanide and barbituric acid could be measured by the FI-straight-line method if the concentration of chloramine-T and pyridine were constant and in sufficient excess. Assuming that cyanide and barbituric acid have a stoichiometry of $m: n$, then the value of $m$ and $n$ can be determined respectively by the proposed FI-straight-line method.

The manifolds shown in Fig. 5 were adopted. When the intermediate was investigated, the reaction coil was $30 \mathrm{~cm}$ long and absorbance was detected at $494 \mathrm{~nm}$. However, the coil length and the detected wavelength were changed to $270 \mathrm{~cm}$ and $580 \mathrm{~nm}$, respectively, when the final product was investigated. Figure $5(\mathrm{~A})$ shows the manifold employed to determine the value of $n$ for producing the intermediate and the final product, in which various concentrations of pyridine-barbituric acid reagent $\left(C_{\mathrm{Barb}}^{0}=\right.$ $0.08-0.2 M$ ) were injected into the system while cyanide was propelled into the system at a constant concentration $\left(C_{\mathrm{CN}-}^{0}=7.6 \times 10^{-5} \mathrm{M}\right)$. Results are shown in Fig. 6, which indicates that the values of $n$ were 1 and 2, respectively, for producing the intermediate and the final product.

From Fig. 6(B) it can be concluded that the reaction molar ratio of cyanide to barbituric acid for producing the final product is $1: 2$, which agrees with results reported in literature. ${ }^{5}$ This fact demonstrates that the FI-straight-line method can be applied to determine the stoichiometry of non-complex reactions.

Although the stoichiometries of cyanide and barbituric acid for producing the intermediate and the final product have been obtained, the reaction mechanism is not clear because the molecular number of cyanide for forming one molecule of the intermediate or the final product is still uncertain. A manifold shown in Fig. 5B was designed to determine the consumed molecular number of cyanide the value of $m$. In this manifold cyanide was injected at various concentrations $\left(C_{\mathrm{CN}-}^{0}=0.2-1.4 \times 10^{-4} M\right)$ while
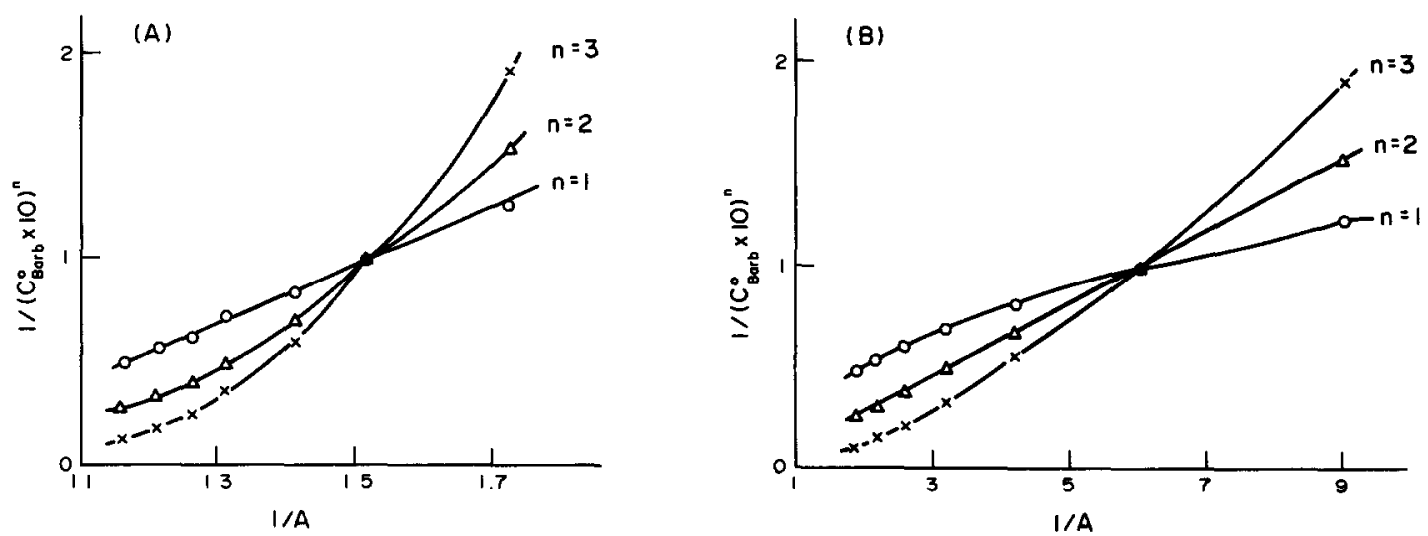

Fig. 6. Determination of the reaction molar ratios of cyanide to barbituric acid for producing the intermediate and the final product by the FI-straight-line method: (A) for investigation of the intermediate; (B) for investigation of the final product. $C_{\text {Barb }}^{0}=0.08-0.2 M ; C_{\mathrm{CN}-}^{0}=7.6 \times 10^{-5} \mathrm{M}$. 

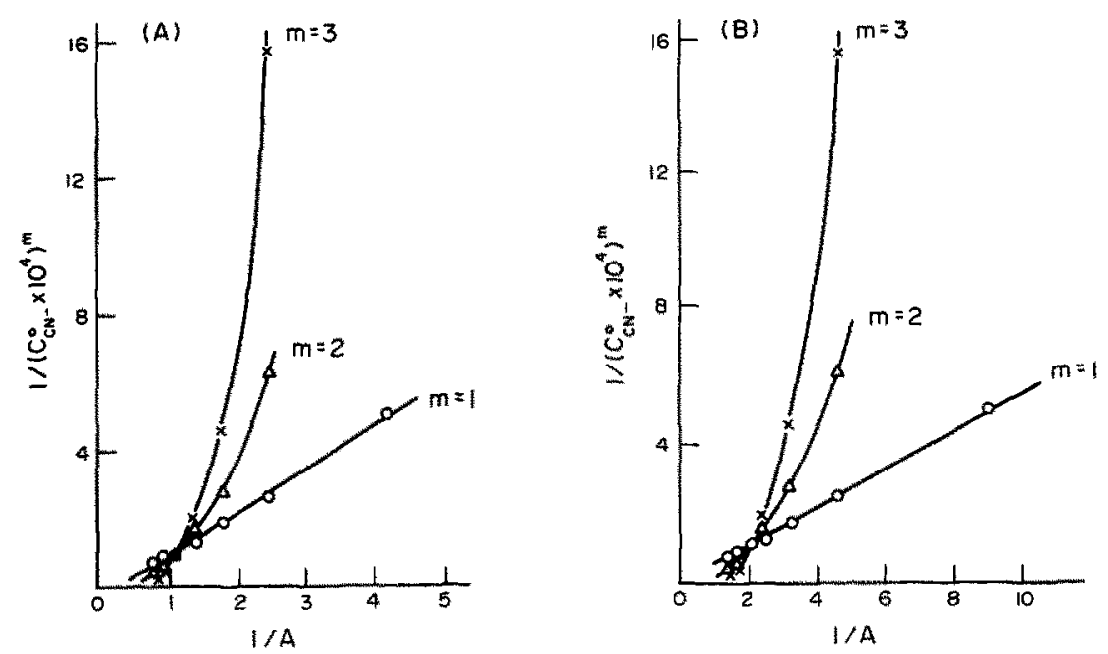

Fig. 7. Determination of the consumed molecular number of cyanide for producing one molecule of the intermediate and the final product by the FI-straight-line method: (A) for investigation of the intermediate; (B) for investigation of the final product. $C_{\mathrm{Barb}}^{0}=0.1 M ; C_{\mathrm{CN}-}^{0}=0.2-1.4 \times 10^{-4} \mathrm{M}$.

barbituric acid was introduced at a constant concentration $\left(C_{\mathrm{Batb}}^{0}=0.1 M\right)$. The results are shown in Fig. 7 which indicates that for both the intermediate and the final product a straight line was obtained by setting $m=1$, i.e. only one molecule of cyanide was consumed for producing one molecule of the intermediate or the final product. As barbituric acid was always in large excess during the measurement of $n$ and $m$, the intermediate and the final product formed during the determination of $n$ must be the same as that formed during the determination of $m$. Therefore, we conclude that for producing one molecule of the intermediate and the final product, the consumed cyanide is one molecule while the consumed barbituric acid is one and two molecules respectively.

\section{CONCLUSIONS}

A new FI-straight-line method was established by combining the Asmus straight-line method with the flow injection technique. The proposed method is suitable for investigating compositions of stable and unstable complexes, especially unstable complexes which cannot be investigated by conventional methods. The method can also be applied to determine stoichiometries of some non-complexing reactions. Since it can be used to measure stoichiometry of reactants when an unstable intermediate is formed, the proposed method has great significance in investigating mechanisms of some complicated reactions.

\section{REFERENCES}

1. E. Asmus, Z. Anal. Chem., 1960, 178, 104.

2. J. Martinez Calatayud, P. Campins Falco and M. C. Pascual Marti, Analyst, 1986, 111, 1317.

3. K. S. Klausen and F. J. Langmyhr, Anal. Chim. Acta, $1963,28,501$.

4. J. Ruzicka and E. H. Hansen, Flow Imjection Analysis, 2nd Edn., p. 29. Wiley, New York, 1988.

5. A. E. Harrey, Jr and D. L. Manning, J. Am. Chem. Soc., $1950,72,4488$.

6. H. Ma and J. Liu, Anal. Chim. Acta, 1992, 261, 247.

7. W. J. Williams, Handbook of Anion Determination. Butterworths, London, 1979. 